\title{
There Are Not Too Many Magic Configurations
}

\author{
Eyal Ackerman • Kevin Buchin • \\ Christian Knauer • Rom Pinchasi • Günter Rote
}

Received: 23 October 2006 / Revised: 22 February 2007 /

Published online: 19 September 2007

(C) Springer Science+Business Media, LLC 2007

\begin{abstract}
A finite planar point set $P$ is called a magic configuration if there is an assignment of positive weights to the points of $P$ such that, for every line $l$ determined by $P$, the sum of the weights of all points of $P$ on $l$ equals 1 . We prove a conjecture of Murty from 1971 and show that if a set of $n$ points $P$ is a magic configuration, then $P$ is in general position, or $P$ contains $n-1$ collinear points, or $P$ is a special configuration of 7 points.
\end{abstract}

Keywords Magic configuration · Euler's formula · Discharging method · Murty's conjecture $\cdot$ Points $\cdot$ Lines $\cdot$ Euclidean plane

\section{Introduction}

Let $P$ be a finite set of points in the plane. $P$ is called a magic configuration if there is an assignment of positive weights to the points of $P$ such that, for every line $l$ determined by $P$, the sum of the weights of all points of $P$ on $l$ equals 1 . Figure 1 shows an example of a point set that is a magic configuration. This special point set (and any projective transformation of it) is called a failed Fano configuration.

The research by Rom Pinchasi was supported by a Grant from the G.I.F., the German-Israeli Foundation for Scientific Research and Development.




Fig. 1 Failed Fano configuration

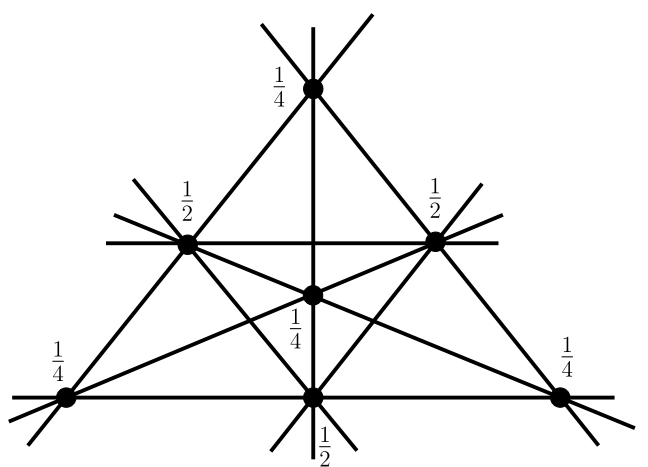

We prove a conjecture of Murty [8] saying that apart from failed Fano configurations, every set of $n$ points that is a magic configuration is either in general position, or contains $n-1$ collinear points. A few other remarks on the history of the problem can be found in The Open Problems Project [2].

Theorem 1 There do not exist magic configurations of cardinality n, other than

- Configurations with n-1 collinear points, or

- Configurations in general position, that is, with no three points on a line, or

- A configuration with 7 points that up to a projective transformation is depicted in Figure 1

We will now make some preliminary observations regarding magic configurations. Many of these observations can be found already in Murty's paper [8].

Assume that a configuration $P$ of $n \geq 2$ points in the plane is magic and that its points are assigned positive weights that witness the fact that $P$ is magic. Recall that a line determined by $P$ is called ordinary if it includes precisely two points of $P$. By Gallai-Sylvester theorem $[4,10]$, the points of $P$ must determine an ordinary line unless they are all collinear.

We claim that unless $P$ has $n-1$ collinear points, then for every point $p \in P$ there is an ordinary line not passing through $p$. Indeed, otherwise, by the theorem of Kelly and Moser [6], the set $P \backslash\{p\}$ determines at least $\frac{3}{7}(n-1)$ ordinary lines (see [1] for the current best bound on the number of ordinary lines determined by $n$ points). Clearly all these lines must be passing through $p$. It follows that at most $\frac{1}{7}(n-1)$ points of $P \backslash\{p\}$ lie on an ordinary line through $p$ and these are all the ordinary lines determined by $P$, contradicting the Kelly-Moser theorem.

It is now easy to see that unless $P$ has $n-1$ collinear points, every point through which there is an ordinary line must be assigned the weight $\frac{1}{2}$. To see this assume that $p$ is such a point and assume without loss of generality that it is assigned a weight that is greater than $\frac{1}{2}$ (otherwise look at the other point on the ordinary line through $p$ ). Let $q$ and $r$ be two points different from $p$ that constitute an ordinary line in $P$. One of $q$ and $r$ is assigned a weight greater than or equal to $\frac{1}{2}$. The sum of the weights assigned to the points on the line through that point and $p$ will be strictly greater than 1 , a contradiction.

Denote by $A$ the set of all points in $P$ through which there is an ordinary line, and assume that $P$ does not have $n-1$ collinear points. Then each point in $A$ is 
Fig. 2 Duality between the plane and the unit sphere



(a) The dual $D(p)$ of a point $p$ is the great circle that is the intersection of $\mathcal{S}$ with the plane through the center of $\mathcal{S}$ that is perpendicular to the line through $p$ and the center of $\mathcal{S}$

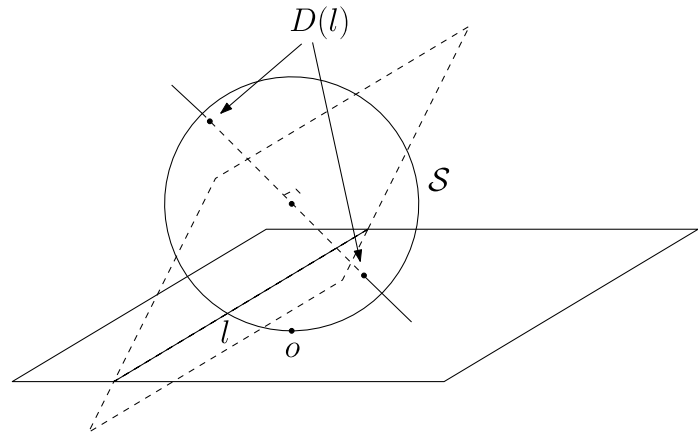

(b) The dual $D(l)$ of a line $l$ is the pair of antipodal points that are the intersection points of $\mathcal{S}$ and the line through the center of $S$ that is perpendicular to the plane through $l$ and the center of $\mathcal{S}$

assigned a weight of $\frac{1}{2}$. It follows that any line through two points in $A$ must be ordinary. Observe that $|A| \geq 3$, as any noncollinear set of points determines at least 3 ordinary lines (see [6]) and this would be impossible if $|A| \leq 2$. Denote by $B$ the set $P \backslash A$. Clearly, every point in $B$ must be assigned a weight that is strictly smaller than $\frac{1}{2}$. Indeed, let $b \in B$ and $a \in A$. The line through $a$ and $b$ cannot be ordinary for otherwise $b \in A$. Therefore it must contain a third point $c$. As the weight assigned to $a$ is $\frac{1}{2}$, it follows that the weight assigned to $b$ can be at most $\frac{1}{2}$ minus the weight assigned to $c$.

Theorem 1 will therefore follow from the following theorem.

Theorem 2 Let $A$ and $B$ be two nonempty sets of distinct points in the Euclidean plane such that $|A| \geq 3$. Assume that all the ordinary lines determined by $A \cup B$ are precisely all the lines determined by two points of A. Assume further that every point in $A \cup B$ is assigned a positive weight such that the sum of the weights of all points on any given line determined by $A \cup B$ is 1 . Then the configuration of points $A \cup B$ is a failed Fano configuration that is equal, up to a projective transformation, to the one shown in Fig. 1, where A consists of the points whose weight is $\frac{1}{2}$. 
Fig. 3 A set of lines that corresponds to the exceptional configuration of Theorem 3



Instead of proving Theorem 2 we will prove its dual theorem on the sphere. We refer here to the standard duality under which the dual $D(p)$ of a point $p$ in the plane is a great circle on the unit sphere $\mathcal{S}$ that touches the plane at the origin. The dual $D(l)$ of a line $l$ in the plane is a pair of antipodal points on $\mathcal{S}$. For a point $p$ in the plane, $D(p)$ is the great circle on $\mathcal{S}$ which is the intersection of $\mathcal{S}$ with the plane through the center of $\mathcal{S}$ that is perpendicular to the line through $p$ and the center of $\mathcal{S}$ (see Fig. 2a). For a line $l$ in the plane, $D(l)$ is the pair of antipodal points that are the intersection points of $\mathcal{S}$ and the line through the center of $S$ that is perpendicular to the plane through $l$ and the center of $\mathcal{S}$ (see Fig. 2b). This duality preserves incidence relations in the sense that if $p$ is a point in the plane that is incident to a line $l$ in the plane, then $D(p)$ is a great circle on $\mathcal{S}$ that is incident to the two points of $D(l)$. Recall that given an arrangement of curves, an ordinary intersection point is an intersection point through which precisely two curves pass.

Theorem 3 Let $A$ and $B$ be two nonempty sets of distinct great circles on a sphere $\mathcal{S}$ such that $|A| \geq 3$. Assume that all the ordinary intersection points determined by $A \cup B$ are precisely all the intersection points determined by A. Assume further that every circle in $A \cup B$ is assigned a positive weight such that the sum of the weights of all circles incident to any given intersection point on $\mathcal{S}$ is 1 . Then the configuration of circles $A \cup B$ is the sphere-dual of a failed Fano configuration that is equal, up to a projective transformation, to the one shown in Fig. 1. The set A consists of the circles dual to the points whose weight is $\frac{1}{2}$.

Figure 3 shows a projection to the plane of the exceptional configuration of Theorem 3. The projection is a central projection through the center of $\mathcal{S}$ on a plane that touches $\mathcal{S}$. Under this projection every two antipodal points on $\mathcal{S}$ are projected to the same point in the plane.

\section{Proof of Theorem 3}

We refer to the circles in $A$ as red circles and to the circles in $B$ as blue circles. We remark that in all the next figures in this paper the solid lines represent the blue circles, while the dashed lines represent the red circles.

For every circle $s \in A \cup B$, let $W(s)$ denote the weight assigned to $s$. As we observed, for every $s \in A$ we have $W(s)=1 / 2$, and for every $s \in B, 0<W(s)<1 / 2$. 
Table 1 Charge of objects of $\mathcal{B}$ before and after Steps $1-4$

\begin{tabular}{|c|c|c|c|c|c|}
\hline object of $\mathcal{B}$ & $\operatorname{ch}(\cdot)$ & $c h_{1}(\cdot)$ & $c h_{2}(\cdot)$ & $\operatorname{ch}_{3}(\cdot)$ & $c h_{4}(\cdot)$ \\
\hline bad crossing point & -1 & 0 & 0 & 0 & 0 \\
\hline good crossing point & $\geq 0$ & $\geq 0$ & $\geq 0$ & $\geq 0$ & $\geq 0$ \\
\hline bad (but not evil) triangle & 0 & 0 & $-1 / 4$ & $\geq 0$ & $\geq 0$ \\
\hline evil triangle & 0 & 0 & $-1 / 4$ & $-1 / 4$ & 0 \\
\hline 0-quadrangle & 1 & 1 & $\geq 0$ & $\geq 0$ & $\geq 0$ \\
\hline 1-quadrangle & 1 & $\geq 0$ & $\geq 0$ & $\geq 0$ & $\geq 0$ \\
\hline good 2-quadrangle & 1 & $\geq 0$ & $\geq 0$ & $\geq 0$ & $\geq 0$ \\
\hline bad 2-quadrangle & 1 & -1 & 0 & 0 & 0 \\
\hline 0 -pentagon & 2 & 2 & $\geq 3 / 4$ & $\geq 0$ & $\geq 0$ \\
\hline 1-pentagon & 2 & $\geq 1$ & $\geq 3 / 4$ & $\geq 1 / 2$ & $\geq 1 / 2$ \\
\hline 2-pentagon & 2 & $\geq 0$ & $\geq 0$ & $\geq 0$ & $\geq 0$ \\
\hline$r$-(k-gon) $, k \geq 6, r \leq\left\lfloor\frac{k}{2}\right\rfloor$ & $k-3$ & $\geq k-3-r$ & $\geq \frac{3}{4} k-3-\frac{r}{2}$ & $\geq \frac{1}{2} k-3$ & $\geq \frac{1}{2} k-3$ \\
\hline
\end{tabular}

We consider the arrangement $\mathcal{B}$ of the circles in $B$ on the sphere $\mathcal{S}$. For every face $f$ in $\mathcal{B}$, the size of $f$ is the number of edges of the face $f$. We will use the term 'triangle' for a face of size three, the term 'quadrangle' for a face of size four, etc. Two faces in $\mathcal{B}$ are called adjacent, if they share an edge. Similarly, two edges in $\mathcal{B}$ are called adjacent, if they are incident to the same crossing point. A great circle $s \in B$ and a face $f$ of $\mathcal{B}$ are called adjacent, if $s$ includes an edge of $f$. We begin by assigning a charge $\operatorname{ch}(\cdot)$ to the faces and vertices of the arrangement $\mathcal{B}$ : The charge of a face of size $k$ is $k-3$, while the charge of a crossing point of exactly $k$ blue circles is $k-3$. For every $k \geq 2$ denote by $f_{k}$ the number of faces in $\mathcal{B}$ of size $k$, and by $t_{k}$ the number of crossing points of exactly $k$ blue circles. It follows from Euler's formula that $\sum_{k}(k-3) f_{k}+\sum_{k}(k-3) t_{k}+6=0$. Therefore, the overall charge is -6 . Observe that any crossing point on a circle $b \in B$, even with a circle in $A$, is a crossing point in $\mathcal{B}$. Indeed, otherwise either it is an ordinary intersection point on $b$, or it is an intersection point that is not ordinary of at least two circles in $A$.

Our plan is to redistribute the charges (discharge) in four steps, such that finally every face and crossing point in $\mathcal{B}$ will have a nonnegative charge. Then it will follow that the total charge is nonnegative, hence a contradiction. For each $i=1,2,3,4$ we will denote by $c h_{i}(\cdot)$ the charge of an object (a face in $\mathcal{B}$ or a crossing point of blue circles) after the $i$ th step. For convenience, Table 1 summarizes the charges of selected objects from $\mathcal{B}$ through the four steps of discharging.

Note that the only elements whose initial charge is negative are crossing points through which there are precisely two blue circles. We call such a crossing point bad. Observe that there are no faces of size two in $\mathcal{B}$. Indeed, otherwise all blue circles pass through the same two antipodal points $p$ and $p^{\prime}$ on the sphere $\mathcal{S}$. As $|A| \geq 3$, there is a circle in $A$ not passing through $p$, and hence also not through its antipodal point $p^{\prime}$. This circle intersects the circles in $B$ in ordinary intersection points, a contradiction.

The following claim and its corollary will be useful throughout the analysis of the discharging steps. 
Fig. $4 d$ is adjacent to two triangles at two of its opposite edges

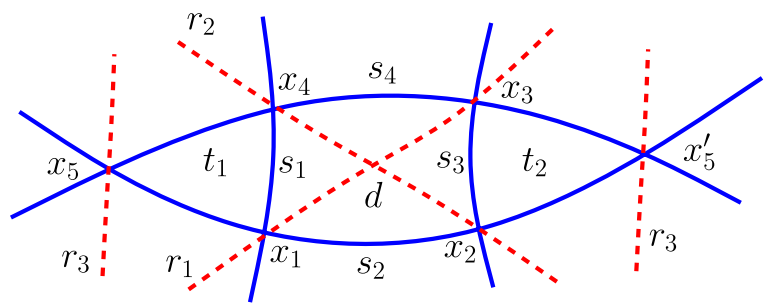

Claim 4 Assume that there is a quadrangle $d$ in $\mathcal{B}$ such that there are precisely two blue circles through every vertex of $d$, and $d$ is adjacent to two triangles at two of its opposite edges. Then $A \cup B$ is the sphere-dual of a failed Fano configuration.

Proof Let $t_{1}$ and $t_{2}$ denote the two triangles adjacent to $d$ at two of its opposite edges. Let $s_{1}, s_{2}, s_{3}$. and $s_{4}$ denote the four blue circles that include the edges of $d$ in the counterclockwise order so that $s_{1}$ and $s_{3}$ separate $d$ from $t_{1}$ and $t_{2}$, respectively. Let $x_{1}, x_{2}, x_{3}$, and $x_{4}$ denote the four vertices of $d$ listed in the counterclockwise order so that $x_{1}$ is the intersection point of $s_{1}$ and $s_{2}$. Since $s_{1}$ and $s_{2}$ are the only blue circles through $x_{1}$ and $s_{4}$ and $s_{1}$ are the only blue circles through $x_{4}$, it follows that $s_{2}$ and $s_{4}$ meet at a vertex of $t_{1}$ that we denote by $x_{5}$. Similarly, $s_{2}$ and $s_{4}$ meet at a vertex of $t_{2}$ that we denote by $x_{5}^{\prime} \cdot x_{5}$ and $x_{5}^{\prime}$ are therefore two antipodal points on the sphere $\mathcal{S}$. Therefore, $x_{1}, x_{2}, x_{5}$ and their antipodal points on $\mathcal{S}$ are the only intersection points on $s_{2}$.

Since there are precisely two blue circles through $x_{1}$, there must be a red circle passing through $x_{1}$. We denote this red circle by $r_{1} . r_{1}$ cannot cross $t_{1}$ and therefore it must cross $d$. Evidently, $r_{1}$ must pass through $x_{3}$. Similarly, there is a red circle $r_{2}$ passing through $x_{2}$ and $x_{4}$. As $|A| \geq 3$, there is a third red circle in $A$ that we denote by $r_{3} . r_{3}$ and $s_{2}$ cannot cross at any other point but $x_{5}$ (and hence also $x_{5}^{\prime}$ ). It follows that there are precisely three red circles in $A$ since a fourth red circle would have to cross $s_{2}$ at a point through which one of $r_{1}, r_{2}$, or $r_{3}$ passes.

We claim that $s_{1}, s_{2}, s_{3}$, and $s_{4}$ are the only blue circles in $B$. Indeed, all other blue circles $s_{5}, \ldots, s_{k}$ must cross $s_{2}$ and $s_{4}$ at $x_{5}$ (and hence also at $x_{5}^{\prime}$ ). None of $r_{3}, s_{5}, \ldots, s_{k}$ can cross $s_{1}$ at $x_{1}$ or $x_{4}$, and only one can cross $s_{1}$ at the intersection point of $s_{1}$ and $s_{3}$. Moreover, no two of $r_{3}, s_{5}, \ldots, s_{k}$ cross $s_{1}$ at a common point. It follows that one of $r_{3}, s_{5}, \ldots, s_{k}$ must cross $s_{1}$ at an ordinary intersection point, a contradiction.

Now it easily follows by inspection that $A \cup B$ must be the sphere-dual of a failed Fano configuration. More specifically, by looking at Fig. 3, we see that the spheredual of the failed Fano configuration has the properties of Claim 4. Moreover, from the assumption of Claim 4 we were led to conclude that there are no lines additional to those drawn in Fig. 4. It is easy to see that the only intersection point (modulo antipodals) not already indicated in Fig. 4 is the common intersection point of $s_{1}, s_{3}$, and $r_{3}$. Hence there is a unique arrangement satisfying the conditions of the claim and it is necessarily the sphere-dual of the failed Fano configuration.

Corollary 5 Assume that $B$ consists of precisely four circles, then $A \cup B$ is the sphere-dual of a failed Fano configuration. 


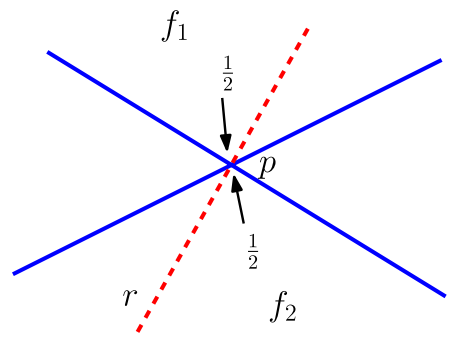

(a) Step 1

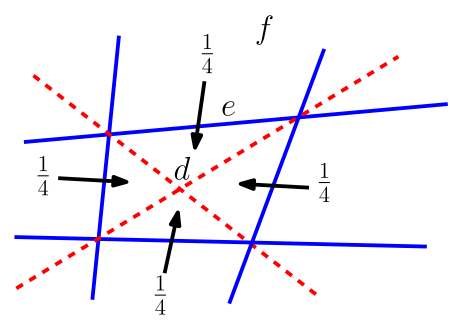

(b) Step 2

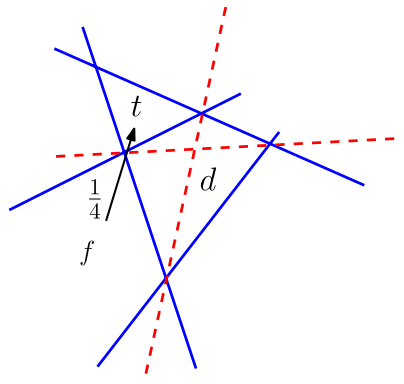

(c) Step 3

Fig. 5 Discharging steps 1-3

Proof By previous arguments not all the circles in $B$ are concurrent. If $B$ has precisely three concurrent circles, then each of them has exactly four crossing points in $\mathcal{B}$. Since the circles in $A$ cross the circles in $B$ only at vertices of $\mathcal{B}$, and $|A| \geq 3$, there must be two circles of $A$ crossing a circle of $B$ at the same point, which is impossible. Therefore, $B$ consists of four circles, no three of which are concurrent. Thus, the arrangement $\mathcal{B}$ satisfies the conditions of Claim 4.

We proceed by describing the four discharging steps and analyzing their effect on the charges of the faces and intersection points of $\mathcal{B}$.

Step 1 (Charging bad crossing points) Let $\mathcal{C}$ denote the arrangement of all circles in $A \cup B$. Since we assume that no ordinary intersection point in $\mathcal{C}$ lies on a blue circle and that every pair of red circles cross at an ordinary point in $\mathcal{C}$, it follows that through each bad crossing point in $\mathcal{B}$ there is precisely one red circle. Let $r$ be a red circle passing through a bad crossing point $p$, and let $f_{1}$ and $f_{2}$ be the two faces in $\mathcal{B}$ that are incident to $p$ and are crossed by $r$ (see Fig. 5a). Then, we take $1 / 2$ units of charge from each of $f_{1}$ and $f_{2}$ and charge it to $p$.

After Step 1 every crossing point of blue circles has a nonnegative charge. Let us now examine the remaining charge at the faces of the arrangement $\mathcal{B}$. A red circle can cross the boundary of a face in $\mathcal{B}$ only at its vertices, for otherwise we would have either an ordinary intersection point of $\mathcal{C}$ on a blue circle, or an intersection point of two (or more) red circles that is not ordinary in $\mathcal{C}$. Thus, every red circle that crosses a face $f$ in $\mathcal{B}$ induces, in fact, a red diagonal in $f$. A face $f$ with $m$ such red diagonals 
Fig. 6 A good 2-quadrangle cannot be incident to exactly one good crossing point

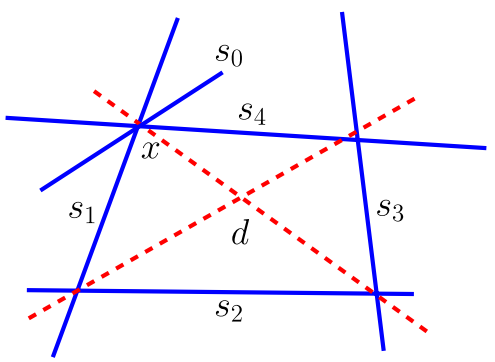

loses at most $m$ units of charge in Step 1. We use an integer before the name of a face in $\mathcal{B}$ to denote the number of its red diagonals. For example, a 2 -hexagon is a face of size six in $\mathcal{B}$ that has precisely two red diagonals. Since triangles cannot have a (red) diagonal, we refer to them simply as 'triangles' instead of 0-triangles. Thus, triangles do not lose charge in Step 1. Pentagons may have at most two red diagonals, and thus they remain with a nonnegative charge as well. The only elements whose charge might be negative after Step 1 are 2-quadrangles, as their charge might be -1 , in case they are incident to four bad crossing points.

A crossing point $x$ of circles from $\mathcal{C}$ is called good, if there is a (necessarily one) red circle through $x$ and at least 3 blue circles through $x$. We call a 2-quadrangle good, if it is incident to a good crossing point. We call a 2-quadrangle that is not incident to any good crossing point a bad 2-quadrangle.

Claim 6 Any good 2-quadrangle is incident to at least two good crossing points.

Proof Assume to the contrary that $d$ is a good 2-quadrangle that is incident to precisely one good crossing point $x$. Let $s_{1}, s_{2}, s_{3}$, and $s_{4}$ denote the four circles in $\mathcal{B}$ that constitute the edges of $d$ in the counterclockwise order so that $s_{1}$ and $s_{4}$ are incident to $x$. As $x$ is a good crossing point, there is another blue circle through $x$ that we denote by $s_{0}$. (See Fig. 6.)

By our assumption, all the crossing points that are incident to $d$, with the exception of $x$, are incident to precisely two blue circles and one red circle. Considering the crossing point of $s_{1}$ and $s_{2}$, we see that $W\left(s_{1}\right)+W\left(s_{2}\right)=1 / 2$. Similarly, considering the crossing point of $s_{2}$ and $s_{3}$, we see that $W\left(s_{2}\right)+W\left(s_{3}\right)=1 / 2$, and in particular $W\left(s_{1}\right)=W\left(s_{3}\right)$. Considering the crossing point of $s_{3}$ and $s_{4}$, we see that $W\left(s_{3}\right)+W\left(s_{4}\right)=1 / 2$. Therefore, $W\left(s_{1}\right)+W\left(s_{4}\right)=W\left(s_{3}\right)+W\left(s_{4}\right)=1 / 2$. But this is a contradiction because considering the circles through $x$ we see that $W\left(s_{1}\right)+W\left(s_{4}\right) \leq 1 / 2-W\left(s_{0}\right)<1 / 2$.

As a corollary of Claim 6, we conclude that after Step 1 every good 2-quadrangle has a nonnegative charge, as it is incident to at most two bad crossing points. We still have to take care of the bad 2-quadrangles. This will be carried out in the next step.

Step 2 (Charging bad 2-quadrangles) In this step every bad 2-quadrangle compensates for its charge shortage by taking $1 / 4$ units of charge from each of its four neighboring faces. That is, let $f$ be a face in $\mathcal{B}$ adjacent to a bad 2-quadrangle $d$, then $d$ 
Fig. 7 A bad triangle cannot be adjacent to two bad quadrangles

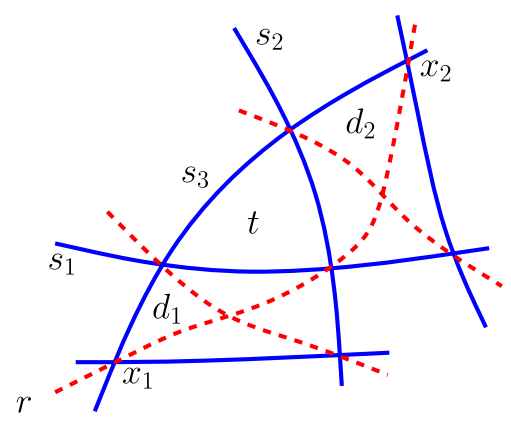

takes the 1/4 units of charge from the charge of $f$ (see Fig. 5b). Note that in such a case $f$ does not have red diagonals at the vertices of the edge common to $f$ and $d$.

It is easy to check, by considering the different possibilities for $f$, that the only elements that might have a negative charge after Step 2 are triangles adjacent to bad 2 -quadrangles. We refer the reader to the proof of Claim 7 for a proof of this observation. We call a triangle that is adjacent to a bad 2-quadrangle a bad triangle. Note that we may assume that a triangle might share an edge with at most one bad 2-quadrangle. Indeed, let $t$ be a triangle adjacent to two bad 2-quadrangles $d_{1}$ and $d_{2}$. Let $s_{1}, s_{2}$, and $s_{3}$ denote the three blue circles that constitute the triangle $t$, such that $s_{1}$ and $s_{2}$ separate $t$ from $d_{1}$ and $d_{2}$, respectively (see Fig. 7).

There is a red circle $r$ through the intersection point of $s_{1}$ and $s_{2} . r$ crosses $s_{3}$ at a vertex $x_{1}$ of $d_{1}$ and at a vertex $x_{2}$ of $d_{2}$, which are therefore antipodal points on the sphere $\mathcal{S}$. It follows that $s_{1}, s_{2}, s_{3}$, and another blue circle that passes through $x_{1}$ and $x_{2}$ are the only blue circles in $B$. By Corollary 5, $A \cup B$ is the sphere-dual of a failed Fano configuration.

Step 3 (Charging some of the bad triangles) In this step we use the excess charge that exists at faces with at least five edges to charge part of the bad triangles.

Let $f$ be a face in $\mathcal{B}$ with $k$ edges, where $k \geq 5$. Let $t$ be a bad triangle adjacent to a bad 2-quadrangle $d$. We transfer $1 / 4$ units of charge from $f$ to $t$, if $f$ and $t$ share a vertex and $f$ is adjacent to (that is, shares an edge with) $d$ (see Fig. 5c).

Before continuing to the last step, we show that after Step 3, every face $f$ with at least five edges remains with a nonnegative charge.

Claim 7 Let $f$ be a face with $k$ edges, where $k \geq 5$. Then after Step $3 f$ has a nonnegative charge.

Proof Let $r$ be the number of red diagonals of $f$. Assume first that $k \geq 6$. Right after Step 1, the charge of $f$ is at least $k-3-r . f$ has exactly $k-2 r$ vertices that are not incident to a red diagonal, and hence at most $k-2 r$ edges none of whose vertices is incident to a red diagonal of $f$. It follows that $f$ may be adjacent to at most $k-2 r$ (bad) 2-quadrangles. Therefore, the charge of $f$ right after Step 2 is at least $k-3-r-\frac{k-2 r}{4}$. As $f$ may contribute $1 / 4$ units of charge to at most $k-2 r$ bad triangles, the charge of $f$ right after Step 3 is at least $k-3-r-\frac{k-2 r}{2}=\frac{k}{2}-3 \geq 0$. 


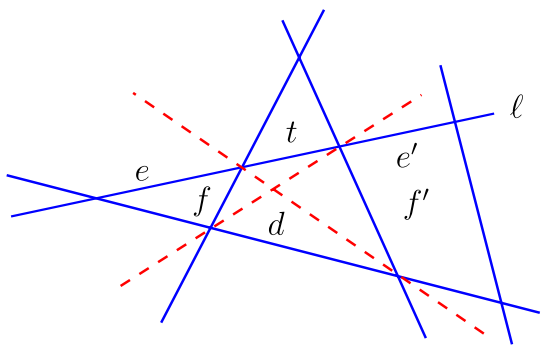

(a) An evil pair $(\ell, d)$ of degree 1 in $H$

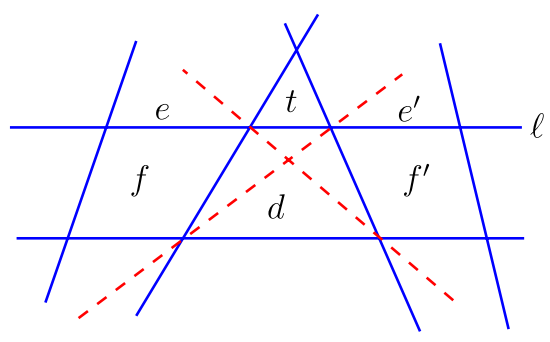

(b) An evil pair $(\ell, d)$ of degree 2 in $H$

Fig. 8 Evil pairs

It is left to consider the case where $f$ is a pentagon. If $f$ is a 2-pentagon, then $f$ cannot be adjacent to any (bad) 2-quadrangle. Therefore, Step 2 as well as Step 3 do not affect the charge of $f$ and it remains at least 0 , as it is right after Step 1. If $f$ is a 1-pentagon, then right after Step 1 the charge of $f$ is at least $1 . f$ may be adjacent to at most one 2-quadrangle. Therefore, right after Step 2 the charge of $f$ is at least 3/4. $f$ contributes $1 / 4$ units of charge in Step 3 to at most one bad triangle and hence remains with a charge of at least $1 / 2$ after Step 3 .

Finally, if $f$ is a 0 -pentagon, then after Step 1 the charge of $f$ is 2 . Observe that if $f$ shares two adjacent edges $e_{1}$ and $e_{2}$ with bad 2-quadrangles $d_{1}$ and $d_{2}$, respectively, then the common vertex of $e_{1}$ and $e_{2}$ cannot be a vertex of a bad triangle $t$. Indeed, otherwise $t$ is adjacent to two bad 2-quadrangles $d_{1}$ and $d_{2}$ which we have previously shown to be only possible in the case where $A \cup B$ is the sphere-dual of a failed Fano configuration. From this observation it follows that if $f$ is adjacent to five bad 2-quadrangles, then it does not share a vertex with any bad triangle and hence the charge of $f$ right after Step 3 is 3/4. If $f$ shares a vertex with five bad triangles, then it may be adjacent to at most two bad 2-quadrangles (in fact one could show that even that is not possible) and hence the charge of $f$ after Step 3 is at least $1 / 4$. In all other cases $f$ is adjacent to at most four bad 2-quadrangles and shares a vertex with at most four bad triangles and hence the charge of $f$ after Step 3 is at least 0 (we remark that this last argument is by far suboptimal, yet suffices for our needs).

Therefore, after Step 3 the only objects with a negative charge are those bad triangles who did not receive 1/4 units of charge in Step 3. We call those triangles evil.

Step 4 (Charging evil triangles) After Step 3 of discharging, the only elements without the desired charge are evil triangles, as they are charged with $-1 / 4$ units of charge each. We will use the excess charge that exists at the 0 -quadrangles to charge with $1 / 4$ units of charge each and every evil triangle.

For every 0-quadrangle $q$, consider the set $E$ of edges of $q$ that are not edges of bad 2-quadrangles. Then the charge of $q$ after Step 3 is $|E| / 4$. For every $e \in E$ let $\ell_{e} \in B$ be the great circle that includes $e$. We call the pair $\left(\ell_{e}, q\right)$ a helping pair and we designate $1 / 4$ unit from the charge of $q$ to the pair $\left(\ell_{e}, q\right)$.

For any evil triangle $t$, let $d$ be the bad 2-quadrangle adjacent to it, and let $\ell \in B$ be the great circle that separates $t$ and $d$. We call the pair $(\ell, d)$ an evil pair. We will 
show that there are at least as many helping pairs as there are evil pairs. Thus we will successfully charge each evil triangle with $1 / 4$ units of charge taken of the excess charge at the 0 -quadrangles after step 3.

Define a bipartite graph $H$ whose vertices are the evil pairs and the helping pairs. Let $(\ell, d)$ be an evil pair, let $t$ be the evil triangle adjacent to $d$ and $\ell$, and let $f$ and $f^{\prime}$ be the two faces in $\mathcal{B}$, other than $t$, that are adjacent to both $\ell$ and $d$. Let $e$ and $e^{\prime}$ be the edges of $f$ and $f^{\prime}$, respectively, on $\ell$. Since $t$ is evil, then $f$ and $f^{\prime}$ can be either triangles or 0-quadrangles (see Fig. 8). Moreover, the edges $e$ and $e^{\prime}$ cannot be edges of bad 2-quadrangles, as $d$ is the only bad 2-quadrangle adjacent to $t$. Each of $(\ell, f)$ and $\left(\ell, f^{\prime}\right)$ is a helping pair, assuming $f$ or $f^{\prime}$, respectively, are not triangles. If $f$ is not a triangle, we connect $(\ell, d)$ in $H$ to the helping pair $(\ell, f)$. Similarly, if $f^{\prime}$ is not a triangle, we connect $(\ell, d)$ in $H$ to the helping pair $\left(\ell, f^{\prime}\right)$. Observe that if both $f$ and $f^{\prime}$ are triangles, then by Claim $4, A \cup B$ is the sphere-dual of a failed Fano configuration. Therefore, we may assume that the degree in $H$ of every evil pair is either 1 or 2 (see Fig. 8). The degree in $H$ of every helping pair is at most 2 , because a helping pair $(\ell, q)$ may be connected only to evil pairs $\left(\ell^{\prime}, d\right)$ such that $\ell=\ell^{\prime}$ and $d$ is adjacent to $q$. It follows that the connected components of $H$ that include evil pairs are either paths alternating between evil pairs and helping pairs, or theoretically, even cycles alternating between evil pairs and helping pairs. Therefore, in order to show that there are at least as many helping pairs as there are evil pairs, it is enough to show that no connected component in $H$ is a path both of whose end vertices are evil pairs.

Indeed, assume to the contrary that there is such a connected component in $H$. Let its vertices be $\left(\ell, d_{1}\right),\left(\ell, q_{1}\right), \ldots,\left(\ell, q_{k-1}\right),\left(\ell, d_{k}\right)$, so that for every $1 \leq i \leq k-1$, $\left(\ell, q_{i}\right)$ is a helping pair connected to both $\left(\ell, d_{i}\right)$ and $\left(\ell, d_{i+1}\right)$. It follows that there is a great circle $\ell^{\prime} \in B$ that includes all edges of $d_{1}, \ldots, d_{k}$ and $q_{1}, \ldots, q_{k-1}$ that are opposite to those included in $\ell$.

Since the degree in $H$ of $\left(\ell, d_{1}\right)$ is 1 , then the face in $\mathcal{B}$, other than $q_{1}$, adjacent to both $\ell, \ell^{\prime}$, and to $d_{1}$ must be a triangle which we denote by $q_{0}$. Similarly, the face in $\mathcal{B}$, other than $q_{k-1}$, adjacent to both $\ell, \ell^{\prime}$, and to $d_{k}$ must be a triangle which we denote by $q_{k}$. Observe that $\ell$ and $\ell^{\prime}$ meet at a vertex of $q_{0}$ and at a vertex of $q_{k}$ (see Fig. 9).

We claim that the only triangles in $\mathcal{B}$ adjacent to $\ell^{\prime}$ are $q_{0}, q_{k}$, and of course their antipodal triangles on the sphere $\mathcal{S}$. This is because for every $0 \leq i \leq k$, the face adjacent to $\ell^{\prime}$ that shares an edge with $q_{i}$ cannot be a triangle as it admits a red diagonal at least at one of its vertices. And moreover, we may assume that for every $1 \leq i \leq k$, the face adjacent to $\ell^{\prime}$ that shares an edge with $d_{i}$ is not a triangle. Indeed, otherwise by Claim 4, $A \cup B$ is the sphere-dual of a failed Fano configuration (recall that there is an evil triangle adjacent to $d_{i}$ on the other side of $\ell$ on $\mathcal{S}$ ). This is a contradiction to a theorem of Levi [7] saying that in any nontrivial arrangement of lines in the real projective plane, every line must be adjacent to at least 3 triangular faces. (Here, we apply Levi's theorem after identifying antipodal points on the sphere $\mathcal{S}$ and thus reducing the great circles in $A \cup B$ to a set of lines in the projective plane.) Since the reference to Levi's theorem is not widely available we refer the reader also to [3, Sect. 5.4] and [5] for very short proofs of Levi's theorem.

We conclude that after Step 4, all the faces in the arrangement $\mathcal{B}$ have a nonnegative charge, and the same holds for every crossing point in $\mathcal{B}$. Thus, the overall charge is nonnegative, contradicting the fact the total charge in the beginning was -6 . 


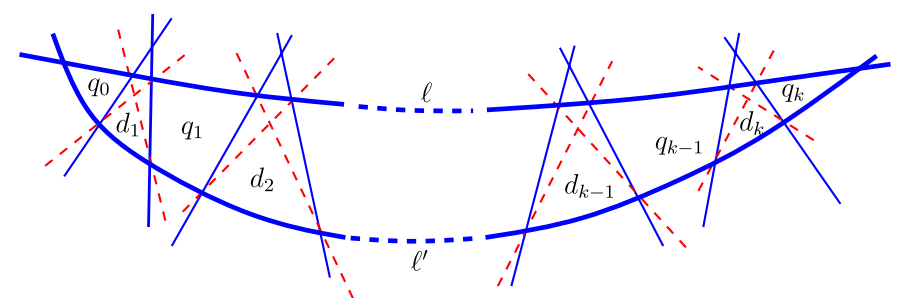

(a)

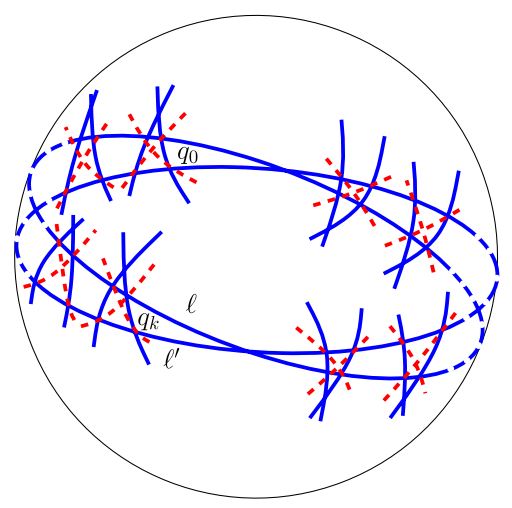

(b)

Fig. 9 A connected component in $H$ both of whose endpoints are evil pairs

\section{Notes and Concluding Remarks}

If the arrangement $\mathcal{B}$ is in general position in the sense that no three blue circle from $B$ pass through the same point, then Theorem 3 and hence also its dual Theorem 2 could be strengthened as follows leaving the proof almost as is:

Theorem 8 Let $A$ and $B$ be two nonempty disjoint sets of points in the plane such that $|A|>1$, and $B$ is in general position. Assume that no line determined by $A$ passes through a point of $B$. Then there is an ordinary line in $A \cup B$ through a point in $B$, unless $A \cup B$ is, up to a projective transformation, the configuration in Fig. 1.

To see why Theorem 8 follows from the proof of Theorem 3, observe that if the circles in $B$ are in general position, then there are no good crossing points in $\mathcal{C}$, and hence the assumptions in Theorem 3 on the weights assigned to the circles in $\mathcal{C}$ are not required. In Theorem 8 we allow more than two points of $A$ to be collinear as long as they are not collinear with a point of $B$. Indeed, in the proof of Theorem 3 we did not really use the assumption that every intersection point determined by $A$ is an ordinary intersection point with respect to $A \cup B$, but only that no intersection point determined by circles from $A$ is incident to a circle from $B$. 
Proving Theorem 8 for the case $B$ is not required to be in general position would imply that the following conjecture ${ }^{1}$ holds. Recall that an ordinary point in a point configuration $P$ is a point $x \in P$ through which there is an ordinary line.

Conjecture 9 Let $G=(V, E)$ be the Sylvester Graph of a finite set of points $P$. That is, $V=\{p \in P \mid p$ is an ordinary point in $P\}$ and $E=\left\{\left(p_{1}, p_{2}\right) \mid p_{1}\right.$ and $p_{2}$ determine an ordinary line in $P\}$. Then $G$ is a complete (nonempty) graph if and only if no three points in $P$ are collinear, or $P$ is a failed Fano configuration.

We would like to note a corollary of Theorem 8 . It is well known that the set of edges of a complete graph on $2 n$ vertices can be partitioned into (necessarily $2 n-1$ ) edge-disjoint perfect matchings. A nice way to realize such a partitioning is to think about the vertices of $K_{2 n}$ as the vertices of a regular $(2 n-1)$-gon plus its center. Then every one of the $2 n-1$ directions of the edges of the $(2 n-1)$-gon induces a perfect matching in which two points are matched if the straight line they determine is parallel to the direction we choose, plus taking the center to be matched with the remaining point. These $2 n-1$ perfect matchings are edge-disjoint.

Now let $G$ be a complete geometric graph on $2 n$ vertices in general position in the plane. We call a matching in $G$ geometrically induced, if the lines containing the edges of the matching are concurrent. If a matching of $G$ is geometrically induced, then the meeting point of all lines that include an edge of the matching is called the center of the matching. The question is can we partition the set of edges of a complete geometric graph $G$ on $2 n$ vertices in general position in the plane into edge-disjoint geometrically induced perfect matchings. By Theorem 8 , this is impossible unless $n=1$ or $n=2$. Indeed, assume it is possible and let $B$ be the set of $2 n$ vertices of $G$, and let $A$ be the set of all points that are the centers of the geometrically induced perfect matchings. Then $A$ and $B$ satisfy the assumptions in Theorem 8 .

It is an interesting open question of what is the maximum possible number of edgedisjoint geometrically induced perfect matchings of a complete geometric graph on $2 n$ vertices in general position in the plane. It seems natural to conjecture that the answer should be $n+1$. This number is attained for the set of vertices of a regular $2 n$-gon in the plane when $n$ is even. Here observe that the geometrically induced perfect matchings whose centers are the points at infinity that correspond to the $n$ directions of the edges of the regular $2 n$-gon plus the center of the $2 n$-gon, are all pairwise edge-disjoint.

One can try to weaken the notion of a magic configuration and omit the restriction of all weights assigned to the points being positive. In this case there seem to be a much larger variety of magic configurations and yet not every configuration is magic. In this context it is interesting to note that given that a configuration is magic (even in the weak sense) it is very easy to assign the right weights (and in a unique way) to the points, just as a function of the number of lines determined by the set that pass through each of the points of the set. To this end let $p_{1}, \ldots, p_{n}$ denote the points of a magic configuration $P$. For every $1 \leq i \leq n$ let $x_{i}$ denote the weight assigned to $p_{i}$ and let $k_{i}$ be the number of lines determined by $P$ that pass through $p_{i}$. For convenience

\footnotetext{
${ }^{1}$ This conjecture is attributed to Sylvester according to Smyth [9].
} 
denote $Y=\sum_{i=1}^{n} x_{i}$. Fix $i$ and consider the point $p_{i}$. There are $k_{i}$ lines determined by $P$ that pass through $p_{i}$. The sum of the weights assigned to the points of $P$ on each of these lines is 1 . It follows that $Y=k_{i}-x_{i}\left(k_{i}-1\right)$. Therefore, $x_{i}=\frac{k_{i}-Y}{k_{i}-1}$. We can get an explicit expression for $x_{i}$ just in terms of $k_{j}(j=1, \ldots, n)$. Observe that $Y=\sum_{j=1}^{n} x_{j}=\sum_{j=1}^{n} \frac{Y-k_{j}}{1-k_{j}}$. Therefore,

$$
Y=\frac{\sum_{j=1}^{n} \frac{k_{j}}{k_{j}-1}}{1+\sum_{j=1}^{n} \frac{1}{k_{j}-1}}, \quad \text { and hence, } \quad x_{i}=\frac{1}{k_{i}-1}\left(k_{i}-\frac{\sum_{j=1}^{n} \frac{k_{j}}{k_{j}-1}}{1+\sum_{j=1}^{n} \frac{1}{k_{j}-1}}\right) .
$$

Observe in particular that if $k_{i}=k_{i^{\prime}}$, then $x_{i}=x_{i^{\prime}}$. It is also clear from here that the weights assignment is unique, if exists.

Acknowledgements We would like to thank Micha Sharir for extremely helpful discussions on Murty's problem. We also thank anonymous referees for several helpful suggestions for improving the presentation of the paper.

\section{References}

1. Csima, J., Sawyer, E.T.: There exist 6n/13 ordinary points. Discrete Comput. Geom. 9(1), 187-202 (1993)

2. Demaine, E., Mitchell, J.S.B., O’Rourke, J.: Problem 65: Magic configurations. The Open Problems Project, http://maven.smith.edu/ orourke/TOPP/P65.html

3. Felsner, S.: Geometric Graphs and Arrangements. Some Chapters from Combinatorial Geometry, 1st edn. Advanced Lectures in Mathematics. Vieweg, Wiesbaden (2004)

4. Gallai, T.: Solution of problem 4065. Am. Math. Mon. 51, 169-171 (1944)

5. Grünbaum, B.: Arrangements and Spreads. Conference Board of the Mathematical Sciences Regional Conference Series in Mathematics, vol. 10. American Mathematical Society, Providence (1972)

6. Kelly, L.M., Moser, W.O.J.: On the number of ordinary lines determined by $n$ points. Can. J. Math. 1, 210-219 (1958)

7. Levi, F.: Die Teilung der projectiven Ebene durch Gerade oder Pseudogerade. Ber. Math.-Phys. Kl. Sächs. Akad. Wiss. 78, 256-267 (1926)

8. Murty, U.S.R.: How many magic configurations are there? Am. Math. Mon. 78(9), 1000-1002 (1971)

9. Smyth, W.F.: Sylvester configurations. James Cook Math. Notes 5-49, 5193-5196 (1989)

10. Sylvester, J.J.: Mathematical question 11851. Educational Times 59, 98-99 (1893) 